\title{
Crystal Structure of Fab Fragment of an Anti-Osteocalcin C-Terminal Peptide Antibody KTM219
}

\author{
Misaki Komatsu ${ }^{1}$, Jinhua Dong ${ }^{2}$, Hiroshi Ueda ${ }^{2},{ }^{\S}$ and Ryoichi Arai ${ }^{1, *}$ \\ ${ }^{1}$ Fac. of Tex. Sci. Tech., Shinshu Univ., Ueda, Nagano 386-8567, Japan \\ ${ }^{2}$ Chemical Resources Lab., Tokyo Inst. of Tech., Yokohama, Kanagawa 226-8503, Japan
}

\section{Introduction}

Recently, we described a novel reagentless fluorescent biosensor strategy named Quenchbody (Q-body), which functions via the antigen-dependent removal of the quenching effect on a fluorophore that is attached to a single-chain antibody variable region [1]. More recently, to explore the practical utility of Quenchbodies, we developed "Ultra-Quenchbody (UQ-body)," antibody Fab fragments that were fluorolabeled at two of the Nterminal regions, and an improved response due to enhanced quenching via dye-dye interactions was observed. An anti-osteocalcin C-terminal peptide antibody KTM219 is one of the antibody Fab fragments suitable for UQ-body. To analyze the detailed structural mechanism of UQ-body and utilize it for further antibody engineering, we have solved the crystal structure of the Fab fragment of an anti-osteocalcin C-terminal peptide antibody KTM219.

\section{Experiments}

The Fab fragment of anti-osteocalcin C-terminal peptide antibody KTM219 was expressed with $\mathrm{His}_{6}$ tag in Escherichia coli, and purified by the following steps: immobilized-metal affinity chromatography, anion exchange chromatography, and gel filtration chromatography. The KTM219 Fab was crystallized at $20^{\circ} \mathrm{C}$ using the hanging drop vapor diffusion method. The KTM219 Fab $(1 \mu \mathrm{L})$ was mixed with the same volume of reservoir solution (0.6 M sodium formate, 29\% PEG 3350). X-ray diffraction data collections were performed at KEK Photon Factory Structural Biology Beamlines at $95 \mathrm{~K}$ with reservoir solution added to $25 \%$ PEG 400 as a cryoprotectant. The structure was solved by molecular replacement method using Phaser with a model structure of anti-emmprin antibody 4A5 Fab (PDB: 4KUZ) [3]. The crystal structure was refined using COOT and REFMAC5.

\section{Results and Discussion}

The KTM219 Fab crystal belongs to the orthorhombic space group $P 2{ }_{1} 22_{1}$, with unit cell constants of $a=64.80$ $\AA, b=71.47 \AA, c=96.88 \AA$, and contains one Fab antibody molecule per asymmetric unit. The structure was refined to $1.9 \AA$ resolution $\left(R_{\text {work }}=19.1 \%, R_{\text {free }}=24.2 \%\right)$. The crystal structure shows that the KTM219 antibody $\mathrm{Fab}$ fragment comprises a light chain $\left(\mathrm{V}_{\mathrm{L}}-\mathrm{C}_{\mathrm{L}}\right)$ and $\mathrm{a}$ heavy chain $\left(\mathrm{V}_{\mathrm{H}}-\mathrm{C}_{\mathrm{H} 1}\right)$ with typical immunoglobulin folds (Fig. 1). A deep pocket forms between $V_{H}$ and $V_{L}$, and it may provide the binding site for the antigen osteocalcin C-terminal peptide (BGP-C7: RRFYGPV). We speculate that the C-terminus of the antigen peptide is inserted into the pocket and the two Arg residues of the antigen may be recognized by the acidic Asp residues on the edge of the entrance of the binding pocket. The present structure of the KTM219 Fab provides useful structural information. To understand the antigen recognition and UQ-body mechanisms of KTM219 in detail, further study is currently in progress.

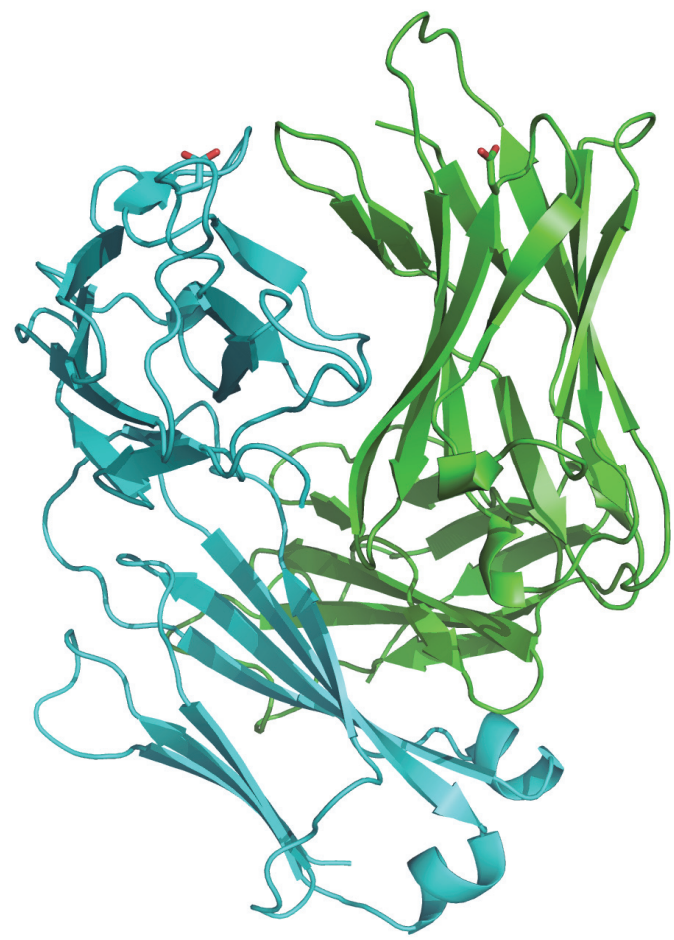

Fig. 1. The crystal structure of the Fab fragment of an anti-osteocalcin C-terminal peptide antibody KTM219. The heavy and light chains are shown in green and cyan, respectively.

\section{$\underline{\text { Acknowledgements }}$}

We thank the PF staff for assistance in X-ray diffraction experiments. This work was supported by JSPS KAKENHI Grant No. 24780097.

\section{References}

[1] R. Abe et al., J. Am. Chem. Soc. 133 (2011) 17386-94.

[2] R. Abe et al., Sci. Rep. 4 (2014) 4640.

[3] A. Teplyakov et al., Proteins 82 (2014) 1679-83.

\footnotetext{
*rarai@shinshu-u.ac.jp

${ }^{\S}$ ueda@res.titech.ac.jp
} 